\title{
On Indoor Visible Light Communication Systems with Spatially Random Receiver
}

Jia $\mathrm{Ye}^{1}$, Gaofeng $\mathrm{Pan}^{1,3}$, Yiyuan $\mathrm{Xie}^{1}$, Quanyuan Feng ${ }^{2}$, Qiang $\mathrm{Ni}^{3}$, and Zhiguo Ding ${ }^{3}$

1. Chongqing Key Laboratory of Nonlinear Circuits and Intelligent Information Processing, Southwest University, Chongqing, 400715, China

2. The School of Information Science \& Technology, Southwest Jiaotong University, Chengdu, 610031, China 3. School of Computing and Communications, Lancaster University, Lancaster, LA1 4WA, U.K

\begin{abstract}
This paper studies the performance of an indoor optical wireless communication system with visible light communication (VLC) technology in a cuboid room with a spatially random receiver. Considering that the receiver is uniformly distributed on the floor of a $4 a \mathrm{~m} \times 4 b \mathrm{~m} \times$ $H \mathrm{~m}$ (where $a>0, b>0$ and $H>0$ ) cuboid room, 4 light emitting diode (LED) lamps are all located at the center of $2 a \mathrm{~m} \times 2 b \mathrm{~m}$ rectangle, which is a quarter of the ceiling area. The receiver chooses the best channel link to receive the information from the LED lamps, which depends on the distance between the receiver and each lamp. By using stochastic geometry theory, we derive the exact/approximated analytical expressions for the outage probability and the ergodic capacity, respectively. Finally, our derived analytical results are verified by Monte Carlo simulations.

Keywords:

Ergodic capacity; outage probability; stochastic geometry; uniformly distributed; visible light communication (VLC)
\end{abstract}

\section{Introduction}

As an age-long technology that can entail the transmission of information-laden optical radiation through the free-space channel [1], optical wireless communications have attracted plenty of attentions of the researchers from both academia and industry due to the features such as good security, free licensed frequency band, immunity to induced electromagnetic interference (EMI), and so on. Among various kinds of optical wireless communication systems, we have seen a growing research in visible light communications(VLC), the idea of which is using light emitting diodes (LEDs) for both illumination and data communications.

LEDs have been demonstrated to have ability to provide a high data rate with considerable energy consumption [2, 3] and to achieve considerable coverage space via the help of relays [4]. Certainly, it's true that VLC has an advantage to transmit radio frequency (RF) signal with less background noise, non-interference, free of health concern and higher security [5, 6, 7]. 
Moreover, some of previous literatures, such as, [8, 9] laid foundations for LED lighting communication systems and contributed to the knowledge base that would provide high data rate and slow growing communication needs, while bringing light to the people or be a decoration.

It is worthwhile to note that one of the features of VLC is the $380 \mathrm{~nm}-780 \mathrm{~nm}$ optical spectrum VLC occupied [10]. Although the frequency band is unlicensed, an appropriate choice for VLC system is given to the indoor VLC due to the fact that the bandwidth is a short-range with limited operating voltage range and coverage. That is to say that, indoor VLCs have an ability to propagate information to the whole room coverage with multiple distributed light emitting diodes, while the LEDs illuminate the room [11]. Furthermore, this is certainly true in the indoor optical wireless system that the channel is typically deterministic.

In addition, intensity modulation (IM)/direct detection (DD) is adopted in indoor VLC systems, which is the most practical modulation technique for indoor scenarios. The receiver would generate electrical signal according to the fluctuations in the intensity carrying the information [12]. Interesting enough, the system also has an ability to obtain a more accurate position information with photo-detector (PD), which could generate photocurrent by the received optical power [13]. Acquiring an accurate indoor localization became more and more possible by the received signal power, as the power of the received optical signal or the received signal is greatly affected by the transmission distance and angles $[13,14,15,16]$.

In addition to position research, many studies on other important indoor systems have been presented. [17] investigated the influence of interference and reflection in VLC indoor system. [18] investigated non-direct line-of-sight (LOS) indoor VLC system performance, which applied repetition code and spatial multiplexing. [19] proposed a new realistic indoor VLC channel model and applied it to multiple-input multiple-output (MIMO) communication system, which uses non-sequential ray tracing approach for the channel impulse responses. [20] illustrated an indoor VLC system with MIMO orthogonal frequency division multiplexing and receiver angular diversity module. [21] proposed centralized or decentralized transmitted power allocation algorithms for multiple input multiple output system considering multiple LEDs and photodetectors. [22] characterized the performance of MIMO systems considering spatial modulation and spatial multiplexing with both imaging and non-imaging receivers. [23] concluded that mobile receivers with adaptive modulation and per antenna rate coding gain higher system capacity compared to the fixed and vertically oriented receivers. [24] demonstrated the superior performance of non-orthogonal multiple access indoor channel. The secrecy outage performance of the VLC downlink was studied in [25], while considering the randomness of the positions of both the legitimate receiver and a group of eavesdroppers. In [26], the secrecy outage performance of the RF uplink was investigated in a VLC-RF hybrid system with light energy harvesting, while considering the randomness of the locations of the legitimate terminal 
and the eavesdropper. A 3-dimensional indoor VLC-RF model with light energy harvesting was proposed and analyzed in [27], while independent and correlated Rician fading channels are considered for the RF uplinks.

\subsection{Motivation and Contributions}

The aforementioned researches $[18,19,20,24]$ only investigated the cases of fixed room sizes $(5 \mathrm{~m} \times 5 \mathrm{~m} \times 3 \mathrm{~m})$ or fixed receiver positions, which result in the fixed distances between the transmitters and the receivers. However, in practice, the viewpoint that the transmission distance and angle largely determine the received power within a same indoor environment is strongly believed. In a more realistic case, a small vary in position will result in a large variation of the received signal, thus we should take the spatial distribution of the receiver and the uncertainty of the room size into consideration. Though [28] considered the random location of the person in the environment and body movements, and [29] took a $W \mathrm{~m} \times L$ $\mathrm{m} \times H \mathrm{~m}$ room into account, they have not derived the closed-form expressions for outage probability (OP).

Motivated by the above observations, in this paper we analyze the performance of an indoor optical wireless communication system with VLC and a randomly distributed receiver by making use of stochastic geometry theory [30]. To investigate the impact of the receiver location, we suppose the receiver is uniformly distributed on the floor of the room with a general size $2 a$ $\mathrm{m} \times 2 b \mathrm{~m} \times H \mathrm{~m}$ (where $a>0, b>0$ and $H>0$ ). In other words, the possibility of falling at each point on the ground in the considered room is the same. It is also important to note here that which point in the room is unknown. For indoor wireless communication structure, considering 4 LED lamps are respectively located at the center of $2 a \mathrm{~m} \times 2 b \mathrm{~m}$ (where $a>0$ and $b>0$ ) rectangle which is a quarter of the ceiling area $4 a \mathrm{~m} \times 4 b \mathrm{~m}$, with the room height, $H \mathrm{~m}(H>0)$. A key concept here is that the receiver only chooses the best channel link among the 4 LEDs to receive the information with the aim of improving channel gain and energy utilization. Main contributions of this work are summarized as follows:

1) We characterize the probability density function (PDF) and cumulative distribution function (CDF) of the signal-to-noise-ratio (SNR) over the VLC channel with two particularities. First, we consider the location of the receiver randomly distributed in the room; Second, the room size is not fixed which is more practical for real scenarios.

2) We study the outage performance of the targeted VLC system and derive the exact closed-form expression for OP.

3) We demonstrate the approximated ergodic capacity for excellent and bad channel environments, which has not been well investigated in the previous works.

In this paper, due to the complexity invoked by the randomness of the terminal's position, 
VLC channel fading and unfixed room size, we employ stochastic geometry and Meijer $G$ functions to achieve performance analysis models.

\subsection{Organization}

The remainder of the paper is organized as follows: The considered indoor VLC system model is given in Section 2. The exact analytical expression for outage probability (OP) is derived in Section 3. The approximate analytical expression for ergodic capacity is presented in Section 4. Numerical results are given in Section 5. Finally, the conclusion will be given in Section 6.

\section{System Model}

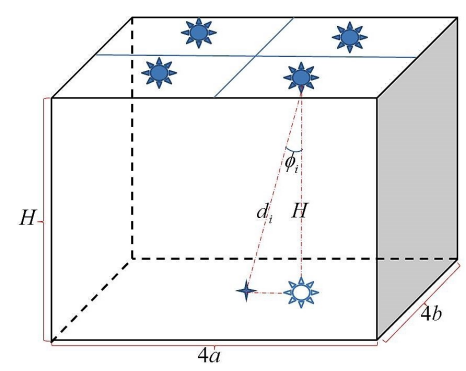

Figure 1: System model

As shown in Fig. 1, an indoor optical wireless communication system with VLC technology is considered here, which consists of four LED lamps placed in the centre of each 1/4 rectangle on the ceiling of the room. All LEDs attempt to deliver information to a receiver equipped with photodiode, which is uniformly distributed on the floor of a $4 a \mathrm{~m} \times 4 b \mathrm{~m} \times H \mathrm{~m}$ room (where $a>0, b>0$ and $H>0$ ). In order to improve the channel gains and energy consumption, we also assume that the receiver would like to receive the strongest information signal emitted by the optimal VLC transmitter among the four LED lamps ${ }^{1}$.

To motivate our discussion about the performance of the system, it is useful to clarify some channel parameters. Due to the fact that the system adopts IM and DD, assuming PD's active area is $A$, the distance between the $i$ th, $(i \in\{1,2,3,4\})$ LED lamp and the receiver is $d_{i}$, the concentrator field of view is $\Psi_{c}$, the gain of the non-imaging concentrator and optical filter adopted at the receiver denoted as $g\left(\psi_{i}\right)$ and $T_{s}\left(\psi_{i}\right)$ respectively, which are related to the angle of incidence with respect to the receiver axis $\psi_{i}$ and defined by Eq. (8) in [31]. $\phi_{i}$ is the

\footnotetext{
${ }^{1}$ In the considered system, each lamp adopts an unique random sequence to encode the information bits, and the four random sequences are orthogonal with each other, which are available at the terminal. Then, after $\mathrm{PD}$, the terminal can respectively decode the four signals from the four lamps by using the random sequences, and compare the SNR of them. In this way, the terminal can find out the signal with the maximum SNR and choose it for information decoding.
} 
LED irradiation angle, while $\phi_{i, 1 / 2}$ is the semi-angle of the LED at half power which determines the light beam Lambertian order $m_{i}$, denoted as

$$
m_{i}=\frac{-\ln 2}{\ln \left(\cos \left(\phi_{i, 1 / 2}\right)\right)} .
$$

Thus, the channel gain between the $i$ th LED lamp and the receiver, $h_{i},(i \in\{1,2,3,4\})$, can be defined as

$$
h_{i}= \begin{cases}\frac{\left(m_{i}+1\right) A}{2 \pi d_{i}^{2}} \cos ^{m_{i}} \phi_{i} T_{s}\left(\psi_{i}\right) g\left(\psi_{i}\right) \cos \psi_{i}, & 0 \leq \psi_{i} \leq \Psi_{c} \\ 0, & \psi_{i}>\Psi_{c}\end{cases}
$$

We can obtain the expression for the received optical power $P_{R i}$ at the receiver, which was transmitted by the $i$ th LED with the same transmit power, $P_{t}$, as

$$
P_{R i}=P_{t} \frac{\left(m_{i}+1\right) A}{2 \pi d_{i}^{2}} \cos ^{m_{i}} \phi_{i} T_{s}\left(\psi_{i}\right) g\left(\psi_{i}\right) \cos \left(\psi_{i}\right) .
$$

As suggested in [32], the RF power constant $C_{R F^{2}}$, including the optical to electrical conversion efficiency, at the receiver follows relationship $\left(P_{R F}\right)^{\frac{1}{2}} \propto i_{P D} \propto P_{r}$, where $i_{P D}$ denotes the current of PD. Thus, the received RF power from the $i$ th LED at the receiver can be written as

$$
P_{i, R F}=C_{R F} P_{R i}^{2}=C_{R F} P_{t}^{2} G_{r}^{2}\left(\phi_{i}\right) G_{t}^{2}\left(\psi_{i}\right) / d_{i}^{4}
$$

where $\psi_{i}=0$ for maximizing the light receiving, which means the incidence line is perpendicular to the PD axis at each receiver. For these reason, we can obtain $G_{r}\left(\psi_{i}\right)=\cos ^{m_{i, r}}\left(\psi_{i}\right)=1$, which is related to light beam Lambertian order $m_{i, r}$. The receiver half power semi-angles $\psi_{i, 1 / 2}$, $m_{i, r}$ can be defined by (1). Similarly, $G_{t}\left(\phi_{i}\right)=\cos ^{m_{i, t}}\left(\phi_{i}\right)$ is the transmitting gain decided by the radiation angle $\phi_{i}$ and $m_{i, t}$, which are related to $\phi_{i, 1 / 2}$ and given by (1). In the following, we also assume that the order $m_{i, t}$ of Lambertian emission for each LED is the same, and denoted as $m_{t}=m_{i, t}$ for simplification.

As shown in Fig. 1, it is obvious that $\cos \left(\phi_{i}\right)=H / d_{i}$. Then, (4) can be rewritten as

$$
\begin{aligned}
P_{i, R F} & =C_{R F} P_{t}^{2} \cos ^{2 m_{t}}\left(\phi_{j}\right) / d_{i}^{4} \\
& =C_{R F} P_{t}^{2} H^{2 m_{t}} d_{i}^{-2 m_{t}-4}
\end{aligned}
$$

In this work, we assume that each LED lamp adopts an unique random sequence to encode the information bits before delivery. Thus, the interference problem among the four LED lights can be overcome, while the four signals from the four lamps can be decoded and distinguished by

\footnotetext{
${ }^{2}$ The second step of Eq. (4) is obtained by substituting Eq. (3) in the first step and the constants of Eq. (3) are absorbed in $C_{R F}$.
} 
using the random sequences. Thus, we only consider the noise at the receiver, while calculating the signal-to-ratio (SNR). Therefore, the SNR of the received signal at the receiver, which is transmitted by the $i$ th transmitter, can be given as

$$
\gamma_{i, R F}=\frac{P_{i, R F}}{N_{0}}=\lambda d_{i}^{-2 m_{t}-4}
$$

where $N_{0}$ is the average power of the noise, $\lambda=C_{R F} P_{t}^{2} H^{2 m_{t}} / N_{0}$ for simplification.

It is important to note here that only the LED with the maximum instantaneous SNR among the four LEDs will be selected. Thus, the final SNR $\gamma_{R F}$ can written as

$$
\gamma_{R F}=\max _{i \in\{1,2,3,4\}}\left\{\gamma_{i, R F}\right\}
$$

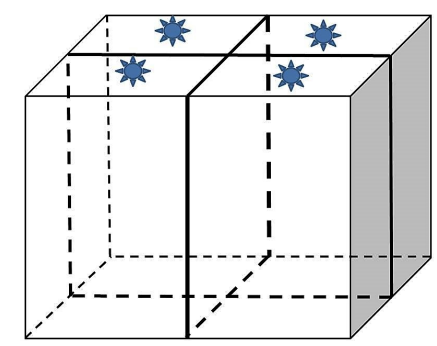

Figure 2: Four sub-rectangles in the room

Observing from the expression of $\gamma_{i, R F}$, apparently, the instantaneous SNR mainly depends on the distance between the receiver and each LED. That is to say, the minimum value of $d_{i}$ will be selected. To better understand this process, let's recall the system model demonstrated by Fig. 1 and divide the whole room into four sub-cubes, where each LED is placed on the center of the ceiling, as presented in Fig. 2. Then, we will see that the LEDs selection problem turns into which sub-rectangle the receiver will be located in. Obviously, the prolixity that the receiver falling on each sub-rectangle depends on their floor area, which are same, $P_{i}=\frac{4 a b}{16 a b}=\frac{1}{4},(i \in\{1,2,3,4\})$ (The four edges that intersect the two rectangles and the center of the room is the special case, where the receiver will receive two or four same intensity of signals and selectively randomly from the transmitters. But the part is only accounting for a small proportion in the total area which will not have a great impact on $\left.P_{i}\right)$. Thus, the CDF of the SNR can be re-expressed as

$$
\begin{aligned}
F_{\gamma_{R F}}(\gamma) & =\sum_{i=1}^{4} P_{i} F_{\gamma_{i}}(\gamma) \\
& =\frac{1}{4} F_{\gamma_{1}}(\gamma)+\frac{1}{4} F_{\gamma_{2}}(\gamma)+\frac{1}{4} F_{\gamma_{3}}(\gamma)+\frac{1}{4} F_{\gamma_{4}}(\gamma)
\end{aligned}
$$

where $F_{\gamma_{i}}(\gamma),(i \in\{1,2,3,4\})$ is the CDF of the SNR for the link between the $i$ th transmitter and the receiver in the $i$ th sub-cube.

To facilitate the following discussion on $\gamma_{R F}$, we establish a direct coordinate system with the center of the lamp projection on the floor in the $i$ th sub-cube, as shown in Fig. 3. Denote 


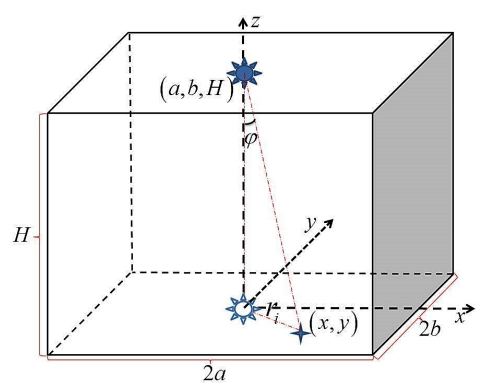

Figure 3: The receiver located in the $i$ th sub-rectangle

the coordinate of the receiver as $(x, y)$. As the receiver is uniformly distributed, the joint probability distribution function of $(x, y)$ can be given as $f_{x y}(x, y)=1 /(4 a b)$. Therefore, the distance within the $i$ th sub-cube between the receiver and the projection of the $i$ th LED can be calculated as $r_{i}=\sqrt{x^{2}+y^{2}}$. Furthermore, we can easily obtain $d_{i}=\sqrt{H^{2}+r_{i}^{2}}$, and write $\gamma_{i}$ as

$$
\gamma_{i}=\lambda\left(H^{2}+r_{i}^{2}\right)^{-m_{t}-2}
$$

where $\gamma_{i, \max }=\lambda H^{-2 m_{t}-4}$ and $\gamma_{i, \min }=\lambda\left(H^{2}+a^{2}+b^{2}\right)^{-m_{t}-2}$.

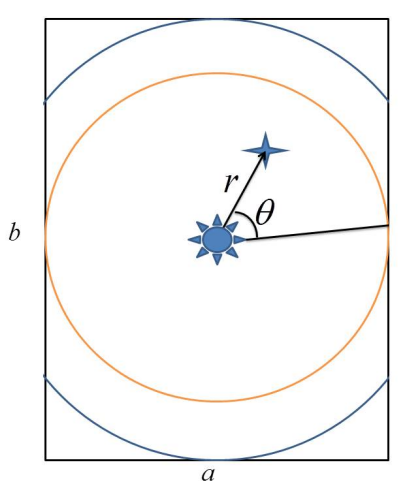

Figure 4: The distribution area of the receiver

In order to obtain the distribution of $r_{i}$, we use polar coordinates measure and assume $a<b$ (For the case of $a>b$, one only needs to turn the sub-cube 90 degrees and let $a=b, b=a$ ) and dividing the rectangle into three parts, as shown in Fig. 4, which will be respectively discussed in the following.

First of all, let's begin with the simplest case that the receiver is within the circular with radius $a$. Under this case, let $z$ denote $r_{i}$ as the independent variable, the CDF and PDF of $r_{i}$ can be easily obtained as

$$
\begin{gathered}
P_{i, 1}(z)=\int_{0}^{2 \pi} \int_{0}^{z} \frac{1}{4 a b} r d r d \theta=\int_{0}^{2 \pi} \frac{z^{2}}{8 a b} d \theta=\frac{\pi z^{2}}{4 a b} \\
f_{i, 1}(z)=\frac{d P_{i, 1}(z)}{d z}=\frac{\pi z}{2 a b}
\end{gathered}
$$


Under the second case that $a<r_{i}<b$, we can respectively obtain the CDF and PDF of $r_{i}$ as

$$
\begin{aligned}
P_{i, 2}(z) & =4\left(\int_{0}^{\arccos \frac{a}{z}} \int_{0}^{\frac{a}{\cos \theta}} \frac{1}{4 a b} r d r d \theta+\int_{\arccos \frac{a}{z}}^{\frac{\pi}{2}} \int_{0}^{z} \frac{1}{4 a b} r d r d \theta\right) \\
& =4\left(\int_{0}^{\frac{\pi}{2}} \frac{a^{2}}{8 a b \cos ^{2} \theta} d \theta+\int_{\arccos \frac{a}{z}}^{\frac{z^{2}}{8 a b}} d \theta\right) \\
& =\frac{z^{2}\left[\pi-2 \arccos \left(\frac{a}{z}\right)\right]}{4 a b}+a \frac{\tan \left[\arccos \left(\frac{a}{z}\right)\right]}{2 b}
\end{aligned}
$$

and

$$
\begin{aligned}
f_{i, 2}(z) & =\frac{d P_{i, 2}(z)}{d z}=\frac{2 z\left[\pi-2 \arccos \left(\frac{a}{z}\right)\right]}{4 a b}-\frac{-2 z^{2}(-a)}{4 a b z^{2} \sqrt{1-\left(\frac{a}{z}\right)^{2}}}+\frac{-a^{2}\left(-z^{-2}\right)}{2 b \cos ^{2}\left[\arccos \left(\frac{a}{z}\right)\right] \sqrt{1-\left(\frac{a}{z}\right)^{2}}} \\
& =\frac{z\left[\pi-2 \arccos \left(\frac{a}{z}\right)\right]}{2 a b} .
\end{aligned}
$$

Similarly, the CDF of $r_{i}$ under the third case that $b<r_{i}<\sqrt{a^{2}+b^{2}}$ can be expressed as

$$
\begin{aligned}
P_{i, 3}(z) & =4\left[\int_{0}^{\operatorname{arc} \cos \frac{a}{z}} \int_{0}^{\frac{a}{\cos \theta}} \frac{1}{4 a b} r d r d \theta+\int_{\arccos \frac{a}{z}}^{\frac{\pi}{2}-\arccos \frac{b}{z}} \int_{0}^{z} \frac{1}{4 a b} r d r d \theta+\int_{\arccos \frac{b}{z}}^{\frac{\pi}{2}} \frac{b}{\cos \theta} \frac{1}{4 a b} r d r d \theta\right] \\
& =4\left[\int_{0}^{\frac{\pi}{2}-\arccos \frac{b}{z}} \frac{a^{2}}{8 a b \cos ^{2} \theta} d \theta+\int_{\arccos \frac{a}{z}}^{\frac{\pi}{2}} \frac{z^{2}}{8 a b} r d r d \theta+\int_{\arccos \frac{b}{z}}^{\frac{b}{z}} \frac{b^{2}}{8 a b \cos ^{2} \theta} d \theta\right] \\
& =\frac{z^{2}\left[\pi-2 \arccos \left(\frac{a}{z}\right)-2 \arccos \left(\frac{b}{z}\right)\right]}{4 a b}+a \frac{\tan \left[\arccos \left(\frac{a}{z}\right)\right]}{2 b}+b \frac{\tan \left[\arccos \left(\frac{b}{z}\right)\right]}{2 a},
\end{aligned}
$$

while the PDF can be written as

$$
\begin{aligned}
f_{i, 3}(z)= & \frac{d P_{i, 3}(z)}{d z} \\
= & \frac{2 z\left[\pi-2 \arccos \left(\frac{a}{z}\right)-2 \arccos \left(\frac{b}{z}\right)\right]}{4 a b}-\frac{2 z^{2}(a)}{4 a b z^{2} \sqrt{1-\left(\frac{a}{z}\right)^{2}}}-\frac{2 z^{2}(b)}{4 a b z^{2} \sqrt{1-\left(\frac{b}{z}\right)^{2}}} \\
& +\frac{a^{2}\left(z^{-2}\right)}{2 b \cos ^{2}\left[\arccos \left(\frac{a}{z}\right)\right] \sqrt{1-\left(\frac{a}{z}\right)^{2}}}+\frac{b^{2}\left(z^{-2}\right)}{2 a \cos ^{2}\left[\arccos \left(\frac{b}{z}\right)\right] \sqrt{1-\left(\frac{b}{z}\right)^{2}}} \\
= & \frac{z\left[\pi-2 \arccos \left(\frac{a}{z}\right)-2 \arccos \left(\frac{b}{z}\right)\right]}{2 a b} .
\end{aligned}
$$

Using [33, Eq. (1.641.1)], $f_{i, 2}(z)$ and $f_{i, 3}(z)$ can be simplified as

$$
f_{i, 2}(z)=\frac{z\left[\arcsin \left(\frac{a}{z}\right)\right]}{a b}
$$

and

$$
f_{i, 3}(z)=\frac{z\left[2 \arcsin \left(\frac{a}{z}\right)+2 \arcsin \left(\frac{b}{z}\right)-\pi\right]}{2 a b},
$$


respectively.

Using (9), we can easily obtain the CDF of $\gamma_{i}$ by

$$
\begin{aligned}
F_{\gamma_{i}}(\gamma) & =\operatorname{Pr}\left\{\gamma_{\mathrm{i}} \leq \gamma\right\}=\operatorname{Pr}\left\{\lambda\left(\mathrm{r}_{\mathrm{i}}^{2}+\mathrm{H}^{2}\right)^{-\mathrm{m}_{\mathrm{t}}-2} \leq \gamma\right\} \\
& =\operatorname{Pr}\left\{\mathrm{r}_{\mathrm{i}} \geq \sqrt{\left(\frac{\gamma}{\lambda}\right)^{-\frac{1}{\mathrm{~m}_{\mathrm{t}}+2}}-\mathrm{H}^{2}}\right\} \\
& =1-\operatorname{Pr}\left\{\mathrm{r}_{\mathrm{i}}<\sqrt{\left(\frac{\gamma}{\lambda}\right)^{-\frac{1}{\mathrm{~m}_{\mathrm{t}+2}}}-\mathrm{H}^{2}}\right\} .
\end{aligned}
$$

Using the obtained CDF of $r_{i}$ and let $\Upsilon=\sqrt{\left(\frac{\gamma}{\lambda}\right)^{-\frac{1}{m_{t}+2}}-H^{2}}$, the CDF of $\gamma_{R F}$ can be rewritten as

$$
F_{\gamma_{\mathrm{i}}}(\gamma)=\left\{\begin{array}{cc}
1-P_{i, 1}(\Upsilon) & 0<\Upsilon<a \\
1-P_{i, 2}(\Upsilon) & a<\Upsilon<b \\
1-P_{i, 3}(\Upsilon) & b<\Upsilon<\sqrt{a^{2}+b^{2}}
\end{array}\right.
$$

Observing the CDF of $r_{i}$, one can have $F_{\gamma_{1}}(\gamma)=F_{\gamma_{2}}(\gamma)=F_{\gamma_{3}}(\gamma)=F_{\gamma_{4}}(\gamma)$. Thus, we can simplify the CDF of $r_{R F}$ as $F_{\gamma_{R F}}(\gamma)=F_{\gamma_{i}}(\gamma)$.

\section{Outage Probability}

In this work, outage probability is defined as the probability that the instantaneous SNR is below a given threshold value, $\gamma_{t h}\left(\gamma_{t h}>0\right)$. Then, the OP of $i$ th link can be given as

$$
\operatorname{Pr}_{\gamma_{\mathrm{i}}}\left(\gamma_{t h}\right)=1-\operatorname{Pr}\left\{\mathrm{r}_{\mathrm{i}}<\sqrt{\left(\frac{\gamma_{\mathrm{th}}}{\lambda}\right)^{-\frac{1}{\mathrm{~m}_{\mathrm{t}}+2}}-\mathrm{H}^{2}}\right\} .
$$

In this paper, as the receiver is uniformly distributed on the indoor floor as suggested by Fig. 4, then, one can easily see that the OP relies on the length and width of the room, and the location of the receiver. Therefore, in the following, we will respectively derive the exact expression of the OP in three cases according to the position of the receiver.

When $0<\sqrt{\left(\frac{\gamma_{t h}}{\lambda}\right)^{-\frac{1}{m_{t}+2}}-H^{2}}<a$, substituting $\Upsilon=\sqrt{\left(\frac{\gamma_{t h}}{\lambda}\right)^{-\frac{1}{m_{t}+2}}-H^{2}}$ into the first formula of (19), the OP of this case can be given as

$$
\operatorname{Pr}_{\gamma_{\mathrm{RF}, 1}}\left(\gamma_{t h}\right)=1-\frac{\pi\left[\left(\frac{\gamma_{t h}}{\lambda}\right)^{-\frac{1}{m_{t}+2}}-H^{2}\right]}{4 a b} .
$$

When $a<\sqrt{\left(\frac{\gamma_{t h}}{\lambda}\right)^{-\frac{1}{m_{t}+2}}-H^{2}}<b$, substituting $\Upsilon=\sqrt{\left(\frac{\gamma_{t h}}{\lambda}\right)^{-\frac{1}{m_{t}+2}}-H^{2}}$ into the second formula of (19), the OP of this case can be given as

$$
\begin{aligned}
\operatorname{Pr}_{\gamma_{\mathrm{RF}, 2}}\left(\gamma_{t h}\right) & =1-a \frac{\tan \left[\arccos \left(a / \sqrt{\left(\frac{\gamma_{t h}}{\lambda}\right)^{-\frac{1}{m_{t}+2}}-H^{2}}\right)\right]}{2 b}-\left[\left(\frac{\gamma_{t h}}{\lambda}\right)^{-\frac{1}{m_{t}+2}}-H^{2}\right] \\
& \times \frac{\left[\pi-2 \arccos \left(a / \sqrt{\left(\frac{\gamma_{t h}}{\lambda}\right)^{-\frac{1}{m_{t}+2}}-H^{2}}\right)\right]}{4 a b} .
\end{aligned}
$$


When $b<\sqrt{\left(\frac{\gamma_{t h}}{\lambda}\right)^{-\frac{1}{m_{t}+2}}-H^{2}}<\sqrt{a^{2}+b^{2}}$, substituting $\Upsilon=\sqrt{\left(\frac{\gamma_{t h}}{\lambda}\right)^{-\frac{1}{m_{t}+2}}-H^{2}}$ into the third formula of (19), the OP of this part can be given as

$$
\begin{aligned}
\operatorname{Pr}_{\gamma_{\mathrm{RF}, 3}}\left(\gamma_{t h}\right)= & 1-a \frac{\tan \left[\arccos \left(a / \sqrt{\left(\frac{\gamma_{t h}}{\lambda}\right)^{-\frac{1}{m_{t}+2}}-H^{2}}\right)\right]}{2 b} \\
& -b \frac{\tan \left[\arccos \left(b / \sqrt{\left(\frac{\gamma_{t h}}{\lambda_{i}}\right)^{-\frac{1}{m_{t}+2}}-H^{2}}\right)\right]}{2 a}-\frac{\left(\frac{\gamma_{t h}}{\lambda}\right)^{-\frac{1}{m_{t}+2}}-H^{2}}{4 a b} \\
\times & {\left[\pi-2 \arccos \left(a / \sqrt{\left(\frac{\gamma_{t h}}{\lambda}\right)^{-\frac{1}{m_{t}+2}}-H^{2}}\right)-2 \arccos \left(b / \sqrt{\left(\frac{\gamma_{t h}}{\lambda}\right)^{-\frac{1}{m_{t}+2}}-H^{2}}\right)\right] . }
\end{aligned}
$$

Therefore, the OP for the considered system can be easily given by the following theorem by using the derived OP for above three cases.

Theorem 1. Assuming an indoor VLC system with a uniformly distributed receiver, the OP of this system can be expressed as

$$
\operatorname{Pr}_{\gamma_{\mathrm{RF}}}\left(\gamma_{t h}\right)=\left\{\begin{array}{cc}
1-\operatorname{Pr}_{\gamma_{\mathrm{RF}, 1}}\left(\gamma_{t h}\right) & 0<\Upsilon<a \\
1-\operatorname{Pr}_{\gamma_{\mathrm{RF}, 2}}\left(\gamma_{t h}\right) & a<\Upsilon<b \\
1-\operatorname{Pr}_{\gamma_{\mathrm{RF}, 3}}\left(\gamma_{t h}\right) & b<\Upsilon<\sqrt{a^{2}+b^{2}}
\end{array} .\right.
$$

Proof. By dividing the rectangle into three parts and using (21), (22) and (23) in (19), Theorem 1 can be easily achieved as (24).

Then, the proof is completed.

\section{Ergodic Capacity}

In this section, we will investigate the ergodic capacity of the target system, which is defined as the expectation value of the instantaneous mutual information between the transmitter and the receiver.

According to the definition, the ergodic capacity can be presented as ${ }^{3}$

$$
C_{R F}(\gamma)=E\left(\log _{2}(1+\gamma)\right)=\frac{1}{\ln 2} \int_{0}^{\infty} \ln (1+\gamma) f_{R F}(\gamma) d \gamma
$$

where $f_{R F}(\gamma)$ is the pdf of the $\gamma_{R F}$, which also equals to $\gamma_{i}$.

\footnotetext{
${ }^{3}$ As suggested in Chapter 6 of [34], the classical Shannon's capacity can work well for the Poisson photon counting channel when rectangular pulse techniques are employed. In this work we present ergodic capacity analysis for the VLC systems adopting rectangular pulse techniques.
} 
Then, using $\gamma_{i}=\lambda\left(H^{2}+r_{i}^{2}\right)^{-m_{t}-2}$, we can caculate $f_{R F}(\gamma)$ as

$$
f_{R F}(\gamma)=-\Upsilon^{\prime} f_{r_{i}}(\Upsilon)
$$

where $\Upsilon^{\prime}$ is the derivative of $\Upsilon$, which can be derived as

$$
\Upsilon^{\prime}=-\frac{\lambda^{\frac{1}{m_{t}+2}} \gamma^{-\frac{m_{t}+3}{m_{t}+2}}}{2\left(m_{t}+2\right) \sqrt{\left(\frac{\gamma}{\lambda}\right)^{-\frac{1}{m_{t}+2}}-H^{2}}}
$$

Thus, by using (11), (16) and (17), the pdf of $\gamma$ can be finally given as

$$
f_{R F}(\gamma)=\left\{\begin{array}{cc}
f_{R F 1}(\gamma)=-\Upsilon^{\prime} f_{i, 1}(\Upsilon) & 0<\Upsilon<a \\
f_{R F 2}(\gamma)=-\Upsilon^{\prime} f_{i, 2}(\Upsilon) & a<\Upsilon<b \\
f_{R F 3}(\gamma)=-\Upsilon^{\prime} f_{i, 3}(\Upsilon) & b<\Upsilon<\sqrt{a^{2}+b^{2}}
\end{array}\right.
$$

Furthermore, the ergodic capacity can be re-expressed as

$$
C_{R F}=\frac{1}{\ln 2}[\underbrace{\int_{\gamma_{a}}^{\gamma_{\max }} \ln (1+\gamma) f_{R F 1}(\gamma) d \gamma}_{I_{1}}+\underbrace{\int_{\gamma_{b}}^{\gamma_{a}} \ln (1+\gamma) f_{R F 2}(\gamma) d \gamma}_{I_{2}}+\underbrace{\int_{\text {min }}^{\gamma_{b}} \ln (1+\gamma) f_{R F 3}(\gamma) d \gamma}_{I_{3}}] .
$$

where $\gamma_{\min }=\gamma_{i, \min }$ and $\gamma_{\max }=\gamma_{i, \max }$ given under $(9)$, while $\gamma_{b}=\lambda\left(H^{2}+b^{2}\right)^{-m_{t}-2}$ and $\gamma_{a}=\lambda\left(H^{2}+a^{2}\right)^{-m_{t}-2}$. In the following, we will calculate $I_{1}, I_{2}$ and $I_{3}$, respectively.

In order to facilitate the following analysis, we first present a useful proposition as follow.

Proposition 1. Defining a new function $g_{1}(A, B, C, D)=\int_{B}^{A} G_{2,2}^{1,2}\left[\left.D x\right|_{1,0} ^{1,1}\right] x^{C-1} d x$, where $A$, $B, C, D$ are constant, $G_{p, q}^{m, n}\left[x \mid \begin{array}{l}a_{1}, \cdots, a_{p} \\ b_{1}, \cdots, b_{q}\end{array}\right]=\frac{1}{2 \pi i} \int \frac{\prod_{j=1}^{m} \Gamma\left(b_{j}-s\right) \prod_{j=1}^{n} \Gamma\left(1-a_{j}+s\right)}{\prod_{j=m+1}^{q} \Gamma\left(1-b_{j}+s\right) \prod_{j=n+1}^{p} \Gamma\left(a_{j}-s\right)} x^{s} d s$ is the Meijer's G-function, as defined by [33, Eq. (9.301)]. Then, we can obtain

$$
g_{1}(A, B, C, D)=A^{C} G_{3,3}^{1,3}\left[D A \mid \begin{array}{l}
1,1+C, 1 \\
1,-C, 0
\end{array}\right]-B^{C} G_{3,3}^{1,3}\left[D B \mid \begin{array}{l}
1,1+C, 1 \\
1,-C, 0
\end{array}\right]
$$

Proof. Making use of [35, Eq. (26)], we can easily calculate $g_{1}(A, B, C, D)$ as (29).

Then, the proof is completed.

To calculate the term $I_{1}$ in (28), we give a lemma as follows.

Lemma 1. Assuming $0<\sqrt{\left(\frac{\gamma_{t h}}{\lambda}\right)^{-\frac{1}{m_{t}+2}}-H^{2}}<a, I_{1}$ can be expressed as

$$
I_{1}=\frac{\pi \lambda^{\frac{1}{m_{t}+2}}}{4\left(m_{t}+2\right) a b} g_{1}\left(\gamma_{\max }, \gamma_{a},-\frac{1}{m_{t}+2}, 1\right)
$$


Proof. It is easy to have

$$
\begin{aligned}
I_{1} & =\int_{\gamma_{a}}^{\gamma_{\max }} \ln (1+\gamma) f_{R F 1}(\gamma) d \gamma \\
& =\int_{\gamma_{a}}^{\gamma_{\max }} \ln (1+\gamma)\left(-\frac{\pi \Upsilon \Upsilon^{\prime}}{2 a b}\right) d \gamma \\
& =\frac{\pi \lambda^{\frac{1}{m_{t}+2}}}{4\left(m_{t}+2\right) a b} \int_{\gamma_{a}}^{\gamma_{\max }} \ln (1+\gamma) \gamma^{-\frac{m_{t}+3}{m_{t}+2}} d \gamma
\end{aligned}
$$

Using [35, Eq.(11)] and $\ln (1+x)=G_{2,2}^{1,2}\left[\left.x\right|_{1,0} ^{1,1}\right]$, (31) can be re-expressed as

$$
I_{1}=\frac{\pi \lambda^{\frac{1}{m_{t}+2}}}{4\left(m_{t}+2\right) a b} \int_{\gamma_{a}}^{\gamma_{\max }} G_{2,2}^{1,2}\left[\gamma \mid \begin{array}{l}
1,1 \\
1,0
\end{array}\right] \gamma^{-\frac{m_{t}+3}{m_{t}+2}} d \gamma
$$

Making use of Proposition 1, we can further obtain $I_{1}$ as (30).

Then, the proof is completed.

Clearly, it is difficult to obtain the exact closed-form expressions for $I_{2}$ and $I_{3}$. In the following, we wil introduce a solution with two simpler cases and derive the approximated results: Case 1: the value of $\lambda$ is relatively high and Case 2: $\lambda$ is relatively low.

\subsection{Case 1: The High $\lambda$ Region}

In subsection, we will derive the expression for the ergodic capacity when $\lambda$ is high, which means a good channel state.

Under this case, large $\lambda$ leads to $\ln \left[1+\lambda\left(t+H^{2}\right)^{-m_{t}-2}\right] \approx \ln \left[\lambda\left(t+H^{2}\right)^{-m_{t}-2}\right]=\ln \lambda-$ $\left(2 m_{t}+4\right) \ln H-\left(m_{t}+2\right) \ln \left(\frac{t}{H^{2}}+1\right)$.

Then, in the following we will respectively derive the approximated expressions for the terms, $I_{2}$ and $I_{3}$, in (28) by giving the following two lemmas.

Lemma 2. Assuming $a<\sqrt{\left(\frac{\gamma_{t h}}{\lambda}\right)^{-\frac{1}{m_{t}+2}}-H^{2}}<b, I_{2}$ in high $\lambda$ region can be expressed as

$$
\begin{aligned}
I_{2, H} & =-\frac{\Omega}{2 a b}\left[\pi a^{2}-b^{2} \arcsin \left(\frac{a}{b}\right)-a^{2} b \sqrt{\frac{1}{a^{2}}-\frac{1}{b^{2}}}\right] \\
& +\frac{m_{t}+2}{2 a b} \sum_{\mathrm{k}=0}^{\infty} \frac{(2 k) ! a^{2 k+1}}{2^{2 k}(k !)^{2}(2 k+1)} g_{1}\left(a^{2}, b^{2},-\frac{2 k-1}{2}, \frac{1}{H^{2}}\right) .
\end{aligned}
$$

Proof. See Appendix A. 
Lemma 3. When $\lambda$ is relatively large, the approximated result for $I_{3, H}$ can be given as

$$
\begin{aligned}
I_{3, H} & =-\frac{\Omega}{2 a b}\left[b^{2} \arcsin \left(\frac{a}{b}\right)+a \sqrt{b-a}-\left(a^{2}+b^{2}\right) \arcsin \left(\frac{a}{\sqrt{a^{2}+b^{2}}}\right)-a b\right]+\frac{m_{t}+2}{2 a b} \\
& \times \sum_{k=0}^{\infty} \frac{(2 k) ! a^{2 k+1}}{2^{2 k}(k !)^{2}(2 k+1)} g_{1}\left(b^{2}, a^{2}+b^{2},-\frac{2 k-1}{2}, \frac{1}{H^{2}}\right) \\
& -\frac{\Omega}{2 a b}\left[\pi b^{2}-\left(a^{2}+b^{2}\right) \arcsin \left(\frac{b}{\sqrt{a^{2}+b^{2}}}\right)-a b\right]+\frac{1}{2 a b} \sum_{k=0}^{\infty} \frac{(2 k) ! b^{2 k+1}\left(m_{t}+2\right)}{2^{2 k}(k !)^{2}(2 k+1)} \\
& \times g_{1}\left(b^{2}, a^{2}+b^{2},-\frac{2 k-1}{2}, \frac{1}{H^{2}}\right)-\frac{\pi \lambda^{\frac{1}{m_{t}+2}}}{4\left(m_{t}+2\right) a b} g_{1}\left(\gamma_{b}, \gamma_{\min },-\frac{1}{m_{t}+2}, 1\right) .
\end{aligned}
$$

Proof. See Appendix B.

Then, when $\lambda$ is relatively large, the ergodic capacity of the target VLC system can be obtained as

$$
C_{R F, H}=\frac{1}{\ln 2}\left\{I_{1}+I_{2, H}+I_{3, H}\right\}
$$

\subsection{Case 2: The Low $\lambda$ Region}

In this subsection, we will derive the expression for the ergodic capacity when $\lambda$ is low, which represents a poor channel state.

Under case 2 , low $\lambda$ leads to $-1<1+\lambda\left(t+H^{2}\right)^{-m_{t}-2}<1$, which satisfies the condition that transforming the logarithmic function into infinite from [33, Eq.(1.151)]

$$
\ln (1+x)=\sum_{n=1}^{\infty}(-1)^{n+1} \frac{x^{n}}{n},(-1<x<1) .
$$

Therefore, in the following we will respectively derive the approximated expressions for the terms, $I_{2}$ and $I_{3}$, in (28) by giving the following two lemmas.

Lemma 4. Assuming $a<\sqrt{\left(\frac{\gamma_{t h}}{\lambda}\right)^{-\frac{1}{m_{t}+2}}-H^{2}}<b, I_{2}$ in low $\lambda$ region can be expressed as

$$
I_{2, \mathrm{~L}}=-\frac{1}{2 a b} \sum_{n=1}^{\infty}(-1)^{n+1} \frac{\lambda^{n}}{n} \sum_{k=0}^{\infty} \frac{(2 k) ! a^{2 k+1}}{2^{2 k}(k !)^{2}(2 k+1)} H^{-(2 m+4) n} g_{2}\left(a^{2}, b^{2},(m+2) n, \frac{2 k+1}{2}\right) .
$$

Proof. See Appendix C.

Lemma 5. Assuming $b<\sqrt{\left(\frac{\gamma_{t h}}{\lambda}\right)^{-\frac{1}{m_{t}+2}}-H^{2}}<\sqrt{a^{2}+b^{2}}, I_{3}$ of this part in low $\lambda$ region can be given as

$$
\begin{aligned}
I_{3, \mathrm{~L}}= & -\frac{1}{2 a b} \sum_{n=1}^{\infty}(-1)^{n+1} \frac{\lambda^{n}}{n} \sum_{k=0}^{\infty} \frac{(2 k) ! a^{2 k+1}}{2^{2 k}(k !)^{2}(2 k+1)} H^{-(2 m+4) n} g_{2}\left(v_{a}, v_{b},(m+2) n, \frac{2 k+1}{2}\right) \\
& -\frac{1}{2 a b} \sum_{n=1}^{\infty}(-1)^{n+1} \frac{\lambda^{n}}{n} \sum_{k=0}^{\infty} \frac{(2 k) ! b^{2 k+1}}{2^{2 k}(k !)^{2}(2 k+1)} H^{-(2 m+4) n} g_{2}\left(v_{a}, v_{b},(m+2) n, \frac{2 k+1}{2}\right) \\
& -\frac{\pi \lambda^{\frac{1}{m_{t}+2}}}{4\left(m_{t}+2\right) a b} g_{1}\left(\gamma_{b}, \gamma_{\min },-\frac{1}{m_{t}+2}, 1\right) .
\end{aligned}
$$


Finally, when $\lambda$ is relatively small, the ergodic capacity of the target VLC system can be obtained as

$$
C_{R F, L}=\frac{1}{\ln 2}\left\{I_{1}+I_{2, L}+I_{3, L}\right\}
$$

\section{Discussion for Multiple Users Scenarios}

In multiple users scenarios where there are multiple terminals share one LED lamp, some orthogonal multiple access (OMA) schemes have been proposed for VLC systems to deal with this issue, e.g., optical orthogonal frequency division multiplexing, single-carrier frequency division multiple access, optical code division multiple access and optical space division multiple access [36]. One can clearly see that under these OMA schemes no interferences exist, and hence a conclusion can be achieved: Our proposed analytical models can work well under these OMA scheme to model and understand the performance of the transmission between the LED lamp and a target terminal.

Recently, non-orthogonal multiple access (NOMA) scheme, which allows all terminals to access the entire bandwidth simultaneously through the principle of power-domain multiplexing, has been introduced into VLC to increase system throughput and accommodate ubiquitous connectivity in VLC systems [36]. Under NOMA scheme and considering the randomness of the positions of the terminals, the performance of the transmissions between the LED lamp and the terminals has not been studied, which is one of the directions for our future works.

\section{Numerical Results}

In this section, we will present the numerical results to validate our proposed analytical models obtained in the previous sections, while the photodetector active area is $A=10^{-4}$, the RF power constant $C_{R F}$ is $7.8 \times 10^{-4}$, the semi-angle of the LED at half power $\phi_{i, 1 / 2}=80^{\circ}$, the gain of optical filter $T_{s}=1$, the transmitted bits and the generated random positions of the receiver both are $1 \times 10^{6}$.

\subsection{Outage Performance}

As observed from Fig. 5, we can see that increasing $a$ and $b$ means increasing the area of the room. Then, the range of the distance between the receiver and the transmitter will be expanded, which finally results in the degraded OP. It is important to note that $a$ and $b$ are the $1 / 4$ length of the sides of the room, which means a small increase in $a$ or $b$ will result in a much higher increase in the area of the floor of the room. 


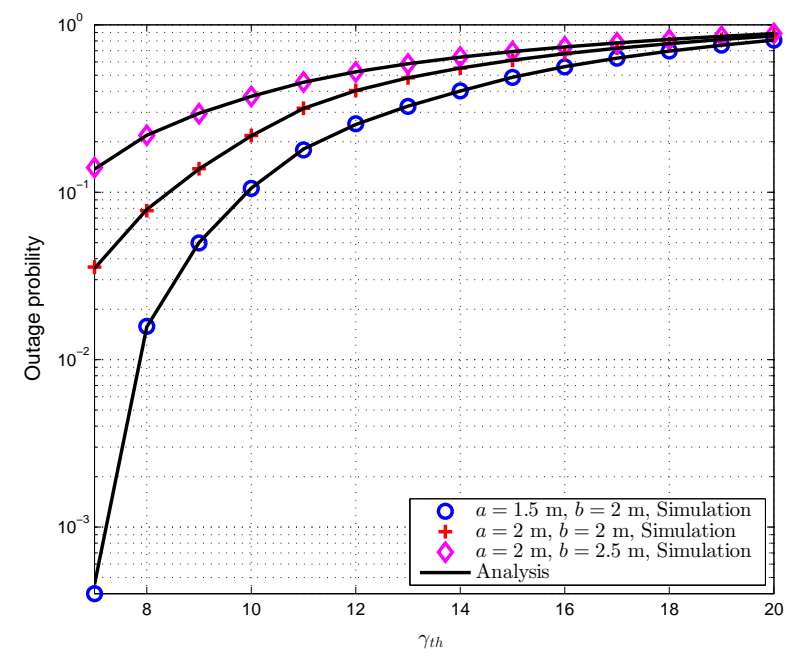

Figure 5: OP versus $\gamma_{t h}$ (the given threshold value) for $P_{t}=5 \mathrm{~W}, N_{0}=-20 \mathrm{~dB}$ and $H=2 \mathrm{~m}$.

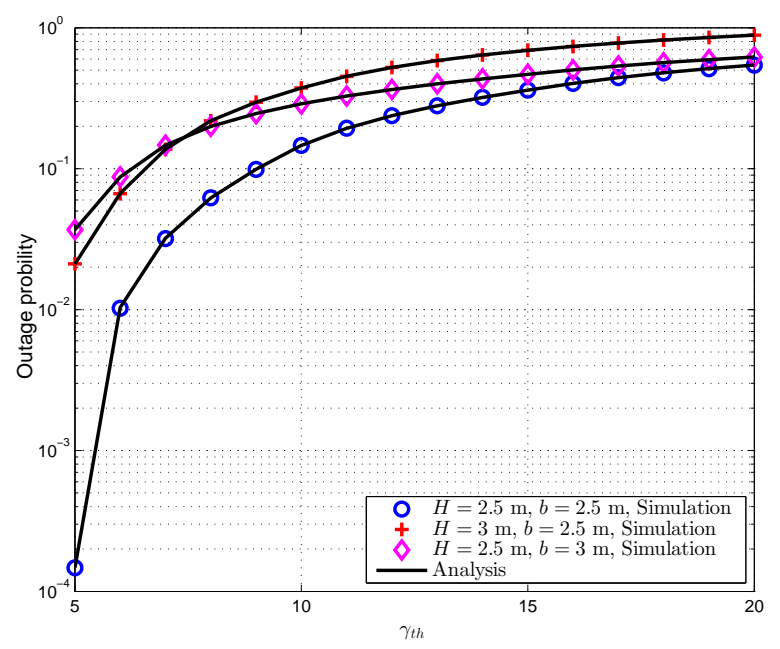

Figure 6: OP versus $\gamma_{t h}$ (the given threshold value) for $P_{t}=5 \mathrm{~W}, N_{0}=-20 \mathrm{~dB}$ and $a=2 \mathrm{~m}$.

As seen from Fig. 6, increasing $H$ also leads to the degraded OP, due to the increased $d_{i}$. Interestingly, $H$ has a greater impact than the ones $b$ or $a$ have, because of the fact that $H$ also effects $\lambda$, which denotes the channel parameter. Comparing the curves of $H=3 \mathrm{~m}, b=2.5 \mathrm{~m}$ and $H=2.5 \mathrm{~m}, b=3 \mathrm{~m}$, we can clearly see that the curve for $H=3 \mathrm{~m}$ will drop slower than the others and will overlap with the curve with a same $b$, as $\gamma_{t h}$ decreases.

Furthermore, we can also see from Figs. 5-6, that the system with a small $\gamma_{t h}$ outperforms the one with a larger $\gamma_{t h}$, since a higher SNR threshold means a better channel situation is needed to achieve the same OP. It is also obvious that simulation and analysis results match very well with each other, which verifies the correctness of our proposed analysis model and the results of Theorem 1. 


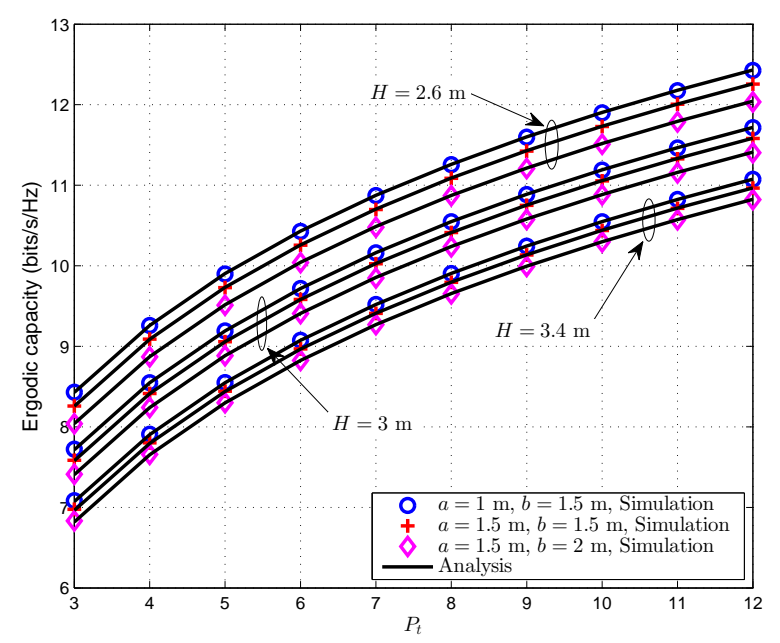

Figure 7: Ergodic capacity versus $P_{t}$ (the same transmit power at all LEDs) for $N_{0}=-35 \mathrm{~dB}$.

\subsection{Ergodic Capacity}

\subsubsection{Case 1: The High $\lambda$ Region}

As shown in Fig. 7, the ergodic capacity for the large $\lambda$ is presented, while the truncation factors of the summation in (33) and (34) are set as 10. Clearly, one can see that decreasing $H$, $a$ and $b$ results in increased ergodic capacity and the height of the room has a greater impact on ergodic capacity compared to $a$ and $b$. This can be explained by the reason for the observation on Fig. 6 in last subsection. It can also be seen from this figure that the channel capacity can be significantly improved by the transmit power $P_{t}$ of the LED, which provides more available received power at the receiver in presence of a same distance.

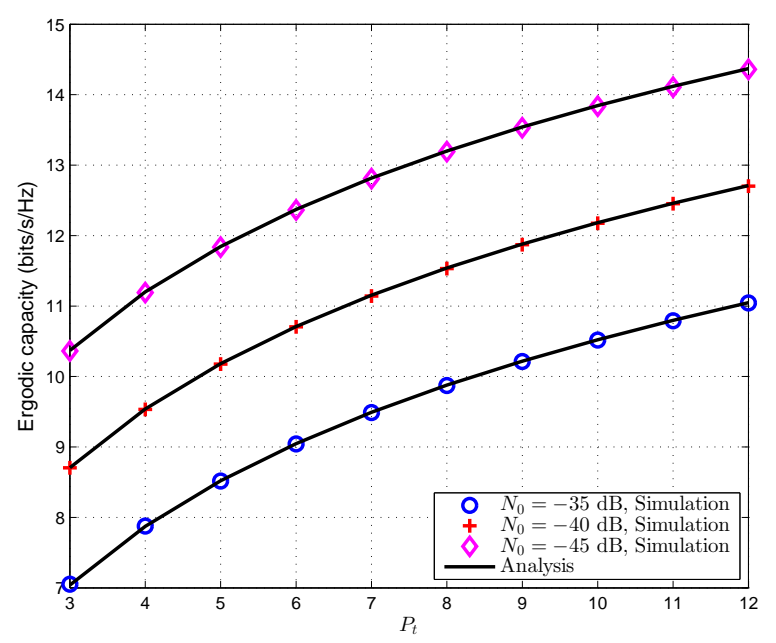

Figure 8: Ergodic capacity versus $P_{t}$ (the same transmit power at all LEDs) for $a=2 \mathrm{~m}, b=2.5 \mathrm{~m}, H=3 \mathrm{~m}$.

Next, we will study the impact of the total noise on the ergodic capacity. As depicted in Fig. 8, it can be clearly observed that ergodic capacity will increase when the power of noise decreases, since the received SNR is improved. It important to note here that the approximate 
results of $\mathbf{L e m \boldsymbol { m a }} \mathbf{5}$ and Lemma $\mathbf{6}$ developed for large $\lambda$ match very well with simulation results.

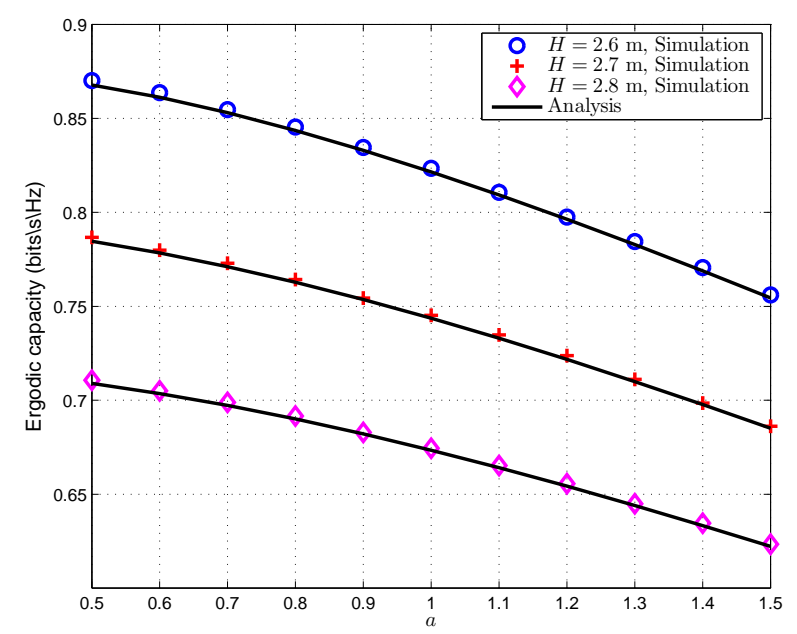

Figure 9: Ergodic capacity versus $a$ for $b=1.5 \mathrm{~m}, P_{t}=7 \mathrm{~W}, N_{0}=20 \mathrm{~dB}$.

\subsubsection{Case 2: The low $\lambda$ Region}

In Fig. 9, the impact of $a$ and $H$ in low $\lambda$ (it includes the transmit power of LEDs, the total channel noise, the height of the room and the $\mathrm{RF}$ power constant) region on the ergodic capacity is presented, while the truncation factors of the summation in (37) and (38) are set as 20 and 10, respectively. It is necessary to note here that the ergodic capacity will be much smaller than the value in the Case 1 due to the condition $-1<1+\lambda\left(t+H^{2}\right)^{-m_{t}-2}<1$. Similar observations can be obtained for the effect of $a$ and $H$ on ergodic capacity as the ones in larger $\lambda$ region. It is clear that the approximated results of $\mathbf{L e m m a ~} \mathbf{7}$ and $\mathbf{L e m m a} \boldsymbol{8}$ are very close to the simulation results and match well in smaller $\lambda$ region. In other words, as observed from the comparison between the expressions for the two cases, we can find that the approximation error of the infinite series for Case 2 is larger than the one of the infinite series for Case 1.

From Figs. 7-9, one can observe that $\lambda$ plays a very important role on the system performance, as it consists of channel parameters, such as room height, RF power constant, the total transmit power, the order of Lambertian emission and noise power. According to the knowledge of the geometric progression, we can reach a conclusion as: the larger truncation factor of the summation leads to the higher approximation accuracy.

\section{Conclusion}

In this paper, the performance of an indoor optical wireless communication system with VLC and a spatially random receiver has been investigated. Specially, the size of the room is unfixed and we only consider selecting the best communication link from four LED lamps 
located at the center of each sub-rectangle. The exact expression for outage probability and the approximated expressions for ergodic capacity have been respectively derived.

The validity of the proposed analytical models has been verified by Monte-Carlo simulations. By observing the numerical results, we can reach the following conclusions:

1) The approximated ergodic capacity can match the simulation ones very well, while assuming relatively large or small $\lambda$, and a large $\lambda$ will lead to better approximation accuracy.

2) The size of the room can significantly influence the performance of the system. More specifically, the height of the room will play a decisive role under a higher outage requirement, while the length and width of the room will be more important with a lower channel threshold value, $\gamma_{t h}$.

3) $\lambda$, which includes the transmit power of LEDs, the total channel noise and the height of the room, can improve the communication quality significantly. This means that the transmit power plays a more important role, compared to two other parameters, since the transmit power plays a positive role while the total channel noise and the height of the room play the negative ones.

\section{Acknowledgment}

This research was supported in part by the National Science Foundation under Grants 61401372, the Fundamental Research Funds for the Central Universities under Grant XDJK2015B023 and XDJK2016A011. The work of Q. Feng was supported by the National Natural Science Foundation of China under Grant 61531016, supported by the project of Science and Technology Support Program of Sichuan Province under Grant 2016GZ0059, and in part by the Sichuan Provincial Science and Technology Important Projects under Grant 2017GZ0110. The work of Z. Ding and Q. Ni were supported by the Royal Society International Exchange Scheme, the UK EPSRC under grant number EP/N005597/1 and EU FP7 CROWN project under grant number PIRSES-GA-2013-610524.

\section{Appendix A: Lemma 2}

According to (28), we can derive $I_{2, H}$ as

$$
\begin{aligned}
I_{2} & =\int_{\gamma_{b}}^{\gamma_{a}} \ln (1+\gamma) f_{R F 2}(\gamma) d \gamma \\
& =\int_{\gamma_{b}}^{\gamma_{a}} \ln (1+\gamma)\left[-\frac{\Upsilon \Upsilon^{\prime} \arcsin \left(\frac{a}{\Upsilon}\right)}{a b}\right] d \gamma \\
& =\frac{1}{2\left(m_{t}+2\right) a b} \int_{\gamma_{b}}^{\gamma_{a}} \ln (1+\gamma) \lambda^{\frac{1}{m_{t}+2}} \gamma^{-\frac{m_{t}+3}{m_{t}+2}} \arcsin \left(a / \sqrt{\left(\frac{\gamma}{\lambda}\right)^{-\frac{1}{m_{t}+2}}-H^{2}}\right) d \gamma
\end{aligned}
$$


To simplify this expression, let $t=\left(\frac{\gamma}{\lambda}\right)^{-\frac{1}{m_{t}+2}}-H^{2}$, we can obtain $\gamma=\lambda\left(t+H^{2}\right)^{-\left(m_{t}+2\right)}$, $d \gamma=-\left(m_{t}+2\right) \lambda\left(t+H^{2}\right)^{-m_{t}-3} d t$ and $\left(\frac{\gamma_{a}}{\lambda}\right)^{-\frac{1}{m_{t}+2}}-H^{2}=a^{2},\left(\frac{\gamma_{b}}{\lambda}\right)^{-\frac{1}{m_{t}+2}}-H^{2}=b^{2}$, we can further derive (40) as

$$
\begin{aligned}
I_{2} & =-\frac{1}{2 a b} \int_{a^{2}}^{b^{2}} \ln \left[1+\lambda\left(t+H^{2}\right)^{-m_{t}-2}\right] \lambda^{\frac{m_{t}+3}{m_{t}+2}}\left[\lambda\left(t+H^{2}\right)^{-m_{t}-2}\right]^{-\frac{m_{t}+3}{m_{t}+2}} \arcsin \left(\frac{a}{\sqrt{t}}\right)\left(t+H^{2}\right)^{-m_{t}-3} d t \\
& =-\frac{1}{2 a b} \int_{a^{2}}^{b^{2}} \ln \left[1+\lambda\left(t+H^{2}\right)^{-m_{t}-2}\right] \arcsin \left(\frac{a}{\sqrt{t}}\right) d t
\end{aligned}
$$

For $\ln \left[1+\lambda\left(t+H^{2}\right)^{-m_{t}-2}\right] \approx \ln \left[\lambda\left(t+H^{2}\right)^{-m_{t}-2}\right]=\ln \lambda-\left(2 m_{t}+4\right) \ln H-\left(m_{t}+2\right) \ln \left(\frac{t}{H^{2}}+1\right)$, $I_{2, H}$ can be approximated as

$$
I_{2, \mathrm{H}} \approx \underbrace{-\frac{1}{2 a b} \int_{b^{2}}^{a^{2}} \Omega \arcsin \left(\frac{a}{\sqrt{t}}\right) d t}_{I_{21}}+\underbrace{\frac{1}{2 a b} \int_{b^{2}}^{a^{2}}\left(m_{t}+2\right) \ln \left(\frac{t}{H^{2}}+1\right) \arcsin \left(\frac{a}{\sqrt{t}}\right) d t}_{I_{22}}
$$

where $\Omega=\ln \lambda-\left(2 m_{t}+4\right) \ln H$ for simplification.

In the following, $I_{21}$ can be derived as

$$
\begin{aligned}
I_{21} & =-\frac{1}{2 a b} \int_{b^{2}}^{a^{2}} \Omega \arcsin \left(\frac{a}{\sqrt{t}}\right) d t \\
& =\frac{1}{\sqrt{t}}-\frac{1}{2 a b} \int_{\frac{1}{b}}^{\frac{1}{a}}-2 u^{-3} \Omega \arcsin (a u) d u \\
& =\frac{1}{a b} \int_{\frac{1}{b}}^{\frac{1}{a}} u^{-3} \Omega \arcsin (a u) d u .
\end{aligned}
$$

Using [37, Eqs.(1.7.3.15)], we can obtain

$$
I_{21}=-\frac{\Omega}{2 a b}\left[\pi a^{2}-b^{2} \arcsin \left(\frac{a}{b}\right)-a^{2} b \sqrt{\frac{1}{a^{2}}-\frac{1}{b^{2}}}\right] .
$$

To derive $I_{2}$, using [33, Eqs.(1.164.1)] to transform the arcsine function into infinite series, one has

$$
\arcsin (x)=\sum_{\mathrm{k}=0}^{\infty} \frac{(2 k) ! x^{2 k+1}}{2^{2 k}(k !)^{2}(2 k+1)}
$$


Further, recalling [35, Eq.(11)] shown above (32) and $g_{1}(A, B, C, D)$, we can derive $I_{22}$ as

$$
\begin{aligned}
I_{22} & =\frac{m_{t}+2}{2 a b} \int_{b^{2}}^{a^{2}} \ln \left(\frac{t}{H^{2}}+1\right) \arcsin \left(\frac{a}{\sqrt{t}}\right) d t \\
& =\frac{m_{t}+2}{2 a b} \int_{b^{2}}^{a^{2}} G_{2,2}^{1,2}\left[\frac{t}{H^{2}} \mid \begin{array}{l}
1,1 \\
1,0
\end{array}\right] \sum_{\mathrm{k}=0}^{\infty} \frac{(2 k) ! a^{2 k+1}}{2^{2 k}(k !)^{2}(2 k+1)} t^{-\frac{2 k+1}{2}} d t \\
& =\frac{m_{t}+2}{2 a b} \sum_{\mathrm{k}=0}^{\infty} \frac{(2 k) ! a^{2 k+1}}{2^{2 k}(k !)^{2}(2 k+1)} \int_{b^{2}}^{a^{2}} G_{2,2}^{1,2}\left[\frac{t}{H^{2}} \mid \begin{array}{l}
1,1 \\
1,0
\end{array}\right] t^{-\frac{2 k+1}{2}} d t \\
& =\frac{m_{t}+2}{2 a b} \sum_{\mathrm{k}=0}^{\infty} \frac{(2 k) ! a^{2 k+1}}{2^{2 k}(k !)^{2}(2 k+1)} g_{1}\left(a^{2}, b^{2},-\frac{2 k-1}{2}, \frac{1}{H^{2}}\right) .
\end{aligned}
$$

Therefore, substituting (44) and (46) into (42), we can obtain the approximated result of $I_{2}$ in high $\lambda$ region.

Then, the proof is completed.

\section{Appendix B: Proof of Lemma 3}

According to (28), we can derive $I_{3}$ as

$$
\begin{aligned}
I_{3} & =\int_{\gamma_{\min }}^{\gamma_{b}} \ln (1+\gamma) f_{R F 3}(\gamma) d \gamma \\
& =-\int_{\gamma_{\min }}^{\gamma_{b}} \ln (1+\gamma) \frac{\Upsilon \Upsilon^{\prime}\left[2 \arcsin \left(\frac{a}{\Upsilon}\right)\right]}{2 a b} d \gamma \int_{\gamma_{\min }}^{\gamma_{b}}\left[\ln (1+\gamma) \frac{\Upsilon \Upsilon^{\prime}\left[2 \arcsin \left(\frac{a}{\Upsilon}\right)\right]}{2 a b}-\ln (1+\gamma) \frac{\Upsilon \Upsilon^{\prime} \pi}{2 a b}\right] d \gamma .
\end{aligned}
$$

Also, substituting $\Upsilon$ and $\Upsilon^{\prime}$ into (47), we can derive $I_{3}$ as

$$
\begin{aligned}
I_{3}= & \underbrace{\frac{\lambda^{\frac{1}{m_{t}+2}}}{2\left(m_{t}+2\right) a b} \int_{\gamma_{\min }}^{\gamma_{b}} \ln (1+\gamma) \gamma^{-\frac{m_{t}+3}{m_{t}+2}} \arcsin \frac{a}{\sqrt{\left(\frac{\gamma}{\lambda}\right)^{-\frac{1}{m_{t}+2}}-H^{2}}} d \gamma}_{I_{31}} \\
& +\underbrace{\frac{\lambda^{\frac{1}{m_{t}+2}}}{2\left(m_{t}+2\right) a b} \int_{\gamma_{\min }}^{\gamma_{b}} \ln (1+\gamma) \gamma^{-\frac{m_{t}+3}{m_{t}+2}} \arcsin \frac{b}{\sqrt{\left(\frac{\gamma}{\lambda}\right)^{-\frac{1}{m_{t}+2}}-H^{2}}} d \gamma}_{I_{32}} \\
& -\underbrace{\frac{\pi \lambda^{\frac{1}{m_{t}+2}}}{4\left(m_{t}+2\right) a b} \int_{\gamma_{\min }}^{\gamma_{b}} \ln (1+\gamma) \gamma^{-\frac{m_{t}+3}{m_{t}+2}} d \gamma}_{I_{33}}
\end{aligned}
$$

To simplify this expression, let $t=\left(\frac{\gamma}{\lambda}\right)^{-\frac{1}{m_{t}+2}}-H^{2}$, we can obtain $\gamma=\lambda\left(t+H^{2}\right)^{-\left(m_{t}+2\right)}$, $d \gamma=-\left(m_{t}+2\right) \lambda\left(t+H^{2}\right)^{-m_{t}-3} d t$ and $\left(\frac{\gamma_{b}}{\lambda}\right)^{-\frac{1}{m_{t}+2}}-H^{2}=b^{2},\left(\frac{\gamma_{\min }}{\lambda}\right)^{-\frac{1}{m_{t}+2}}-H^{2}=a^{2}+b^{2}$, we can further derive $I_{31}$ and $I_{32}$ of (48) as 


$$
\begin{aligned}
I_{31} & =-\frac{1}{2 a b} \int_{b^{2}}^{a^{2}+b^{2}} \ln \left[1+\lambda\left(t+H^{2}\right)^{-m_{t}-2}\right] \lambda^{\frac{m_{t}+3}{m_{t}+2}}\left[\lambda\left(t+H^{2}\right)^{-m_{t}-2}\right]^{-\frac{m_{t}+3}{m_{t}+2}} \arcsin \left(\frac{a}{\sqrt{t}}\right)\left(t+H^{2}\right)^{-m_{t}-3} d t \\
& =-\frac{1}{2 a b} \int_{b^{2}}^{a^{2}+b^{2}} \ln \left[1+\lambda\left(t+H^{2}\right)^{-m_{t}-2}\right] \arcsin \left(\frac{a}{\sqrt{t}}\right) d t
\end{aligned}
$$

and

$$
\begin{aligned}
I_{32} & =-\frac{1}{2 a b} \int_{b^{2}}^{a^{2}+b^{2}} \ln \left[1+\lambda\left(t+H^{2}\right)^{-m_{t}-2}\right] \lambda^{\frac{m_{t}+3}{m_{t}+2}} \\
& \cdot\left[\lambda\left(t+H^{2}\right)^{-m_{t}-2}\right]^{-\frac{m_{t}+3}{m_{t}+2}} \arcsin \left(\frac{b}{\sqrt{t}}\right)\left(t+H^{2}\right)^{-m_{t}-3} d t \\
& =-\frac{1}{2 a b} \int_{b^{2}}^{a^{2}+b^{2}} \ln \left[1+\lambda\left(t+H^{2}\right)^{-m_{t}-2}\right] \arcsin \left(\frac{b}{\sqrt{t}}\right) d t .
\end{aligned}
$$

Similar to the approximate method of the (42), (49) and (50) can be further derived as

$$
\begin{aligned}
I_{31}= & -\frac{1}{2 a b} \int_{a^{2}+b^{2}}^{b^{2}} \ln \left[1+\lambda_{i j}\left(t+H^{2}\right)^{-m_{t}-2}\right] \arcsin \left(\frac{a}{\sqrt{t}}\right) d t \\
\approx & -\frac{1}{2 a b} \int_{a^{2}+b^{2}}^{b^{2}} \Omega \arcsin \left(\frac{a}{\sqrt{t}}\right) d t+\frac{1}{2 a b} \int_{a^{2}+b^{2}}^{b^{2}}\left(m_{t}+2\right) \ln \left(\frac{t}{H^{2}}+1\right) \arcsin \left(\frac{a}{\sqrt{t}}\right) d t \\
= & \frac{1}{a b} \int_{\frac{1}{a+b}}^{\frac{1}{b}} u^{-3} \Omega \arcsin (a u) d u+\frac{m_{t}+2}{2 a b} \sum_{\mathrm{k}=0}^{\infty} \frac{(2 k) ! a^{2 k+1}}{2^{2 k}(k !)^{2}(2 k+1)} \int_{a^{2}+b^{2}}^{b^{2}} G_{2,2}^{1,2}\left[\frac{t}{H^{2}} \mid \frac{1,1}{1,0}\right] t^{-\frac{2 k+1}{2}} d t \\
= & -\frac{\Omega^{2}}{2 a b}\left[b^{2} \arcsin \left(\frac{a}{b}\right)+a \sqrt{b-a}-\left(a^{2}+b^{2}\right) \arcsin \left(\frac{a b]+\frac{m_{t}+2}{2 a b}}{\sqrt{a^{2}+b^{2}}}\right)-a b\right. \\
& \times \sum_{k=0}^{\infty} \frac{(2 k) ! a^{2 k+1}}{2^{2 k}(k !)^{2}(2 k+1)} g_{1}\left(b^{2}, a^{2}+b^{2},-\frac{2 k-1}{2}, \frac{1}{H^{2}}\right)
\end{aligned}
$$

and

$$
\begin{aligned}
I_{32}= & -\frac{1}{2 a b} \int_{b^{2}}^{a^{2}+b^{2}} \ln \left[1+\lambda\left(t+H^{2}\right)^{-m_{t}-2}\right] \lambda^{\frac{m_{t}+3}{m_{t}+2}} \\
\approx & -\frac{1}{2 a b} \int_{a^{2}+b^{2}}^{b^{2}} \Omega \arcsin \left(\frac{b}{\sqrt{t}}\right) d t+\frac{1}{2 a b} \int_{a^{2}+b^{2}}^{b^{2}}\left(m_{t}+2\right) \ln \left(\frac{t}{H^{2}}+1\right) \arcsin \left(\frac{b}{\sqrt{t}}\right) d t \\
= & \frac{1}{a b} \int_{\frac{1}{a+b}}^{\frac{1}{b}} u^{-3} \Omega \arcsin (b u) d u+\frac{m_{t}+2}{2 a b} \sum_{k=0}^{\infty} \frac{(2 k) ! b^{2 k+1}}{2^{2 k}(k !)^{2}(2 k+1)} \int_{a^{2}+b^{2}}^{b^{2}} G_{2,2}^{1,2}\left[\frac{t}{H^{2}} \mid 1,1\right] t^{-\frac{2 k+1}{2}} d t \\
= & -\frac{\Omega}{2 a b}\left[\pi b^{2}-\left(a^{2}+b^{2}\right) \arcsin \left(\frac{b}{\sqrt{a^{2}+b^{2}}}\right)-a b\right]+\frac{1}{2 a b} \\
& \times \sum_{k=0}^{\infty} \frac{(2 k) ! b^{2 k+1}\left(m_{t}+2\right)}{2^{2 k}(k !)^{2}(2 k+1)} g_{1}\left(b^{2}, a^{2}+b^{2},-\frac{2 k-1}{2}, \frac{1}{H^{2}}\right)
\end{aligned}
$$


Comparing $I_{33}$ to $I_{1}$, one can find that the difference between them is the upper and lower limits of the integral. Thus, we can easily obtain

$$
\begin{aligned}
I_{33} & =\frac{\pi \lambda^{\frac{1}{m_{t}+2}}}{4\left(m_{t}+2\right) a b} \int_{\gamma_{\min }}^{\gamma_{b}} \ln (1+\gamma) \gamma^{-\frac{m_{t}+3}{m_{t}+2}} d \gamma \\
& =\frac{\pi \lambda^{\frac{1}{m_{t}+2}}}{4\left(m_{t}+2\right) a b} \int_{\gamma_{\min }}^{\gamma_{b}} G_{2,2}^{1,2}\left[\gamma \mid \begin{array}{l}
1,1 \\
1,0
\end{array}\right] \gamma^{-\frac{m_{t}+3}{m_{t}+2}} d \gamma \\
& =\frac{\pi \lambda^{\frac{1}{m_{t}+2}}}{4\left(m_{t}+2\right) a b} g_{1}\left(\gamma_{b}, \gamma_{\min },-\frac{1}{m_{t}+2}, 1\right)
\end{aligned}
$$

Substituting (51), (52) and (53) into (48), we can obtain $I_{3, H}$ as Lemma 6.

Then, the proof is completed.

\section{Appendix C: Proof of Lemma 4}

Using (36) and (45) to transform the logarithmic function and arcsine function into infinite series, we can derive (41) as

$$
\begin{aligned}
I_{2, \mathrm{~L}} & =-\frac{1}{2 a b} \int_{b^{2}}^{a^{2}} \sum_{n=1}^{\infty}(-1)^{n+1} \frac{\left[\lambda\left(t+H^{2}\right)^{-m-2}\right]^{n}}{n} \sum_{k=0}^{\infty} \frac{(2 k) !}{2^{2 k}(k !)^{2}(2 k+1)}\left(\frac{a}{\sqrt{t}}\right)^{2 k+1} d t \\
& =-\frac{1}{2 a b} \int_{b^{2}}^{a^{2}} \sum_{n=1}^{\infty}(-1)^{n+1} \frac{\lambda^{n}}{n} \sum_{k=0}^{\infty} \frac{(2 k) !}{2^{2 k}(k !)^{2}(2 k+1)} a^{2 k+1}\left(t+H^{2}\right)^{-(m+2) n} t^{-\frac{2 k+1}{2}} d t \\
& =-\frac{1}{2 a b} \sum_{n=1}^{\infty}(-1)^{n+1} \frac{\lambda^{n}}{n} \sum_{k=0}^{\infty} \frac{(2 k) ! a^{2 k+1}}{2^{2 k}(k !)^{2}(2 k+1)} H^{-(2 m+4) n} \int_{b^{2}}^{a^{2}}\left(\frac{t}{H^{2}}+1\right)^{-(m+2) n} t^{-\frac{2 k+1}{2}} d t .
\end{aligned}
$$

To calculate the integral in last equation, we give a useful proposition as follows.

Proposition 2. Defining another new function $g_{2}(A, B, C, D)=\int_{B}^{A}\left(\frac{t}{H^{2}}+1\right)^{-C} t^{-D} d x$, we can rewrite $g_{2}(A, B, C, D)$ as

$$
g_{2}(A, B, C, D)=G_{2,2}^{1,2}\left[\frac{A}{H^{2}} \mid \begin{array}{c}
1-C, D \\
0, D-1
\end{array}\right] \frac{A^{-D+1}}{\Gamma(C)}-G_{2,2}^{1,2}\left[\frac{B}{H^{2}} \mid \begin{array}{l}
1-C, D \\
0, D-1
\end{array}\right] \frac{B^{-D+1}}{\Gamma(C)}
$$

Proof. Making use of [38, Eq. (8.4.2.5)], we can rewrite $g_{2}(A, B, C, D)$ as

$$
g_{2}(A, B, C, D)=\int_{B}^{A} G_{1,1}^{1,1}\left[\left.\frac{t}{H^{2}}\right|_{0} ^{1-C}\right] \frac{t^{-D}}{\Gamma(C)} d t
$$

Therefore, (55) can be obtained by using [35, Eq. (26)].

Then, the proof is finished.

Thus, using Proposition 2, the expression of $I_{2, L}$ can be obtained.

Then, the proof is completed. 


\section{Appendix D: Proof of Lemma 5}

Obviously, the derivation of $I_{3, L}$ will be similar with the ones of $I_{1}$ and $I_{2, L}$. Thus, we can obtain

$$
\begin{aligned}
I_{3, \mathrm{~L}}= & -\frac{1}{2 a b} \int_{a^{2}+b^{2}}^{b^{2}} \sum_{n=1}^{\infty}(-1)^{n+1} \frac{\left[\lambda\left(t+H^{2}\right)^{-m-2}\right]^{n}}{n} \sum_{k=0}^{\infty} \frac{(2 k) !}{2^{2 k}(k !)^{2}(2 k+1)}\left(\frac{a}{\sqrt{t}}\right)^{2 k+1} d t \\
& -\frac{1}{2 a b} \int_{a^{2}+b^{2}}^{b^{2}} \sum_{n=1}^{\infty}(-1)^{n+1} \frac{\left[\lambda\left(t+H^{2}\right)^{-m-2}\right]^{n}}{n} \sum_{k=0}^{\infty} \frac{(2 k) !}{2^{2 k}(k !)^{2}(2 k+1)}\left(\frac{b}{\sqrt{t}}\right)^{2 k+1} d t \\
& -\frac{\pi \lambda^{\frac{1}{m_{t}+2}}}{4\left(m_{t}+2\right) a b} \int_{\gamma_{\min }}^{\gamma_{b}} \ln (1+\gamma) \gamma^{-\frac{m_{t}+3}{m_{t}+2}} d \gamma .
\end{aligned}
$$

Using Proposition 2, the expression of $I_{3, L}$ can be easily obtained.

Then, the proof is completed.

\section{References}

[1] F. R. Gfeller and U. Bapst, "Wireless in-house data communication via diffuse infrared radiation," in Proc. IEEE., vol. 67, no. 11, pp. 1474-1486, Nov. 1979.

[2] D. Tsonev et al., "A 3-Gb/s single-LED OFDM-based wireless VLC link using a gallium nitride LED," IEEE Photon. Technol. Lett., vol. 26, no. 7, pp. 637-640, Apr. 2014.

[3] H. Chun et al., "Visible light communication using a black GaN LED and fluorescent polymer color converter," IEEE Photon. Technol. Lett., vol. 26, no. 20, pp. 2035-2038, Oct. 2014.

[4] C. Zhang, J. Ye, G. Pan and Z. Ding, "Cooperative hybrid VLC-RF systems with spatially random terminals," to appear in IEEE Trans. Commun., DOI: 10.1109/TCOMM.2018.2865949.

[5] J. Grubor, S. Randel, K. D. Langer and J. W. Walewski, "Broadband information broadcasting using LED-based interior lighting," J. Lightw. Technol., vo1. 26, no. 24, pp. 3883-3892, Dec. 2008.

[6] H. Elgala, R. Mesleh, and H. Haas, "Indoor optical wireless communication: Potential and state-of-the-art," IEEE Commun. Mag., vol. 49, no. 9, pp. 56-62, Sept. 2011.

[7] H. Haas, "High-speed wireless networking using visible light," SPIE Newsroom., 2013.

[8] R. X. G. Ferreira et al., "High bandwidth GaN-based micro-LEDs for multi-Gb/s visible light communications," IEEE Photon. Technol. Lett., vol. 28, no. 19, pp. 2023-2026, Oct. 2016. 
[9] H. Marshoud, V. M. Kapinas, G. K. Karagiannidis et al., "Non-orthogonal multiple access for visible light communications," IEEE Photon. Technol. Lett., vol. 28, no. 1, pp. 51-54, Jan. 2016.

[10] Y. Zheng and M. Zhang, "Visible light communications-recent progresses and future outlooks," in Proc. SOPO, Chengdu, China, 2010, pp. 1-6.

[11] H. Burchardt, N. Serafimovski, D. Tsonev, S. Videv and H. Haas, "VLC: Beyond pointto-point communication," IEEE Commun. Mag., vol. 52, no. 7, pp. 98-105, Jul. 2014.

[12] J. Armstrong, "OFDM for optical communications," J. Lightw. Technol., vol. 27, no. 3, pp. 189-204, Feb. 2009.

[13] H. S. Kim, D. R. Kim, S. H. Yang, Y. H. Son and S. K. Han, "An indoor visible light communication positioning system using a RF carrier allocation technique," J. Lightw. Technol., vol. 31, no. 1, pp. 134-144, Jan. 2013.

[14] J. Yan and B. Zhu, "A visible light communication indoor localization algorithm in rotated environments," in Proc. CITS, Kunming, China, 2016, pp. 1-4.

[15] S. Lee, S. Y. Jung, "Location awareness using angle-of-arrival based circular-PD-array for visible light communication," in Proc. APCC, Jeju, South Korean, 2012, pp. 480-485.

[16] S. H. Yang, and S. K. Han, "VLC based indoor positioning using single-Tx and rotatable single-Rx," in Proc. COIN, Jeju, South Korean, 2014, pp. 1-2.

[17] T. Komine and M. Nakagawa, "Fundamental analysis for visible-light communication system using LED lights," IEEE Trans. Consum. Electron., vol. 50, no. 1, pp. 100-107, Feb. 2004.

[18] N. A. Tran, D. A. Luong, T. C. Thang and A. T. Pham, "Performance analysis of indoor MIMO visible light communication systems," in Proc. ICCE, Danang, Vietnam, 2014, pp. 60-64.

[19] A. Yesilkaya, F. Miramirkhani, E. Basar, E. Panayirci and M. Uysal, "Performance of MIMO enhanced unipolar OFDM with realistic indoor visible light channel models," in Proc. IEEE WCNC, Doha, Qatar, 2016, pp. 1-6.

[20] Y. Hong, T. Wu and L. K. Chen, "On the performance of adaptive MIMO-OFDM indoor visible light communications," IEEE Photon. Technol. Lett., vol. 28, no. 8, pp. 907-910, Apr. 2016. 
[21] J. Lian and M. Brandt-Pearce, "Multiuser MIMO Indoor Visible Light Communication System Using Spatial Multiplexing," J. Lightw. Technol., vol. 35, no. 23, pp. 5024-5033, Dec. 2017.

[22] P. M. Butala, H. Elgala and T. D. C. Little, "Performance of optical spatial modulation and spatial multiplexing with imaging receiver," in Proc. IEEE WCNC, Istanbul, Turkey, 2014, pp. 394-399.

[23] P. Fahamuel, J. Thompson and H. Haas, "Improved indoor VLC MIMO channel capacity using mobile receiver with angular diversity detectors," in Proc. IEEE GCC, Austin, USA, 2014, pp. 2060-2065.

[24] R. C. Kizilirmak, C. R. Rowell and M. Uysal, "Non-orthogonal multiple access (NOMA) for indoor visible light communications," in Proc. IWOW, Istanbul, Turkey, 2015, pp. 98-101.

[25] G. Pan, J. Ye, Z. Ding, "On secure VLC systems with spatially random terminals," IEEE Commun. Lett., vol. 21, no. 3, pp. 492-495, Mar. 2017.

[26] G. Pan, J. Ye, Z. Ding, "Secure hybrid VLC-RF systems with light energy harvesting," IEEE Trans. Commun., vol. 65, no. 10, pp. 4348-4359, Oct. 2017.

[27] G. Pan, H. Lei, Z. Ding, Q. Ni, "On 3-D hybrid VLC-RF systems with light energy harvesting and OMA scheme over RF link," in Proc. IEEE GLOBECOM, Singapore, Dec. 2017, pp. 4-8.

[28] C. Le Bas, S. Sahuguede, A. Julien-Vergonjanne, A. Behlouli, P. Combeau and L. Aveneau, "Impact of receiver orientation and position on visible light communication link performance," in Proc. IWOW, Istanbul, Turkey, 2015, pp. 1-5.

[29] Wu D, Ghassemlooy Z, Zhong W D, et al. "Effect of optimal Lambertian order for cellular indoor optical wireless communication and positioning systems," Opt. Eng., 2016.

[30] M. Haenggi, Stochastic Geometry for Wireless Networks., 2012.

[31] J. M. Kahn and J. R. Barry, "Wireless infrared communications," Proc. IEEE., vol. 85, no. 2, pp. 265-298, Feb. 1997.

[32] S. H. Yang, E. M. Jung and S. K. Han, "Indoor location estimation based on LED visible light communication using multiple optical receivers," IEEE Commu. Lett., vol. 17, no. 9, pp. 1834-1837, Sep. 2013.

[33] I. S. Gradshteyn and I. M. Ryzhik, Table of Integrals, Series, and Products, 7th edition. Academic Press, 2007. 
[34] S. Hranilovic, Wireless Optical Communication Systems, Springer-Verlag New York, Inc., New York, NY, 2009.

[35] V. S. Adamchik and O.I. Marichev, "The algorithm for calculating integrals of hypergeometric type functions and its realization in REDUCE system," in Proc. ISSAC 1990., Tokyo, Japan pp. 212-224, Aug. 1990.

[36] S. S. Bawazir, P. C. Sofotasios, S. Muhaidat, Y. Al-Hammadi and G. K. Karagiannidis, "Multiple access for visible light communications: Research challenges and future trends," IEEE Access, vol. 6, pp. 26167-26174, 2018.

[37] A. P. Prudnikov, Yu. A. Brychkov, and O. I. Marichev, Integrals and Series: vol. 1, Elementary Functions, 3rd Ed., New York: Gordon \& Breach Sci. Publ., 1992.

[38] A.P. Prudnikov, Y.A. Brychkov, and O.I. Marichev, Integrals and Series: Vol. 3: More Special Functions, New York: CRC Press, 1992. 